\title{
SAFETY RELATED MISCONCEPTIONS AND SELF-REPORTED BEHAVIORAL ADAPTATIONS ASSOCIATED WITH ADVANCED IN-VEHICLE SYSTEMS: LESSONS LEARNED FROM EARLY TECHNOLOGY ADOPTERS
}

\author{
Robert E. Llaneras \\ Virginia Tech Transportation Institute \\ Blacksburg, VA USA \\ E-mail: ellaneras@vtti.vt.edu
}

\begin{abstract}
Summary: Early adopters of advanced in-vehicle technologies (Adaptive Cruise Control, night vision, park aid, and navigation systems) were interviewed in an effort to assess the extent to which drivers come to understand the performance capabilities and limitations of these types of advanced systems, and to understand how systems are influencing driver behavior (modifying behavior in potentially positive or negative ways). Despite access to a wide array of information about their in-vehicle system, responses to knowledge-based questions about the systems themselves suggest that key information was not necessarily acquired nor understood by a large number of drivers. Many drivers held misconceptions about the performance capabilities of their advanced systems, suggesting that drivers' mental models of how these systems function and perform do not always match reality. For example, $99 \%$ of ACC system owners did not know that the system ignores stopped vehicles. Similarly, $41 \%$ of park aid system owners did not know that the system warning is tied solely on the distance to objects and does not take into account their closing speed. Self-reported data also provided evidence of behavioral adaptations. Results suggest that additional efforts are needed to increase driver understanding of how these systems operate, particularly for safety-related aspects.
\end{abstract}

\section{INTRODUCTION}

Previous work has highlighted the importance of ensuring that drivers understand the functional limitations of driver support systems, and the safety consequences likely to occur when drivers lack full understanding of automation (Seppelt \& Lee, 2007; Nilsson, 1995). Early adopters of advanced in-vehicle technologies, currently available in production automobiles (Adaptive Cruise Control, night vision, park aid, and navigation systems), were interviewed in an effort to understand how these types of systems are influencing driver behavior (modifying behavior in potentially positive or negative ways) and to assess the extent to which drivers accept these advanced systems and come to understand their performance capabilities and limitations. The specific research objectives undertaken as part of this work included:

- Assessing driver understanding of advanced in-vehicle technologies.

- Identifying potential driver behavioral adaptations.

- Determining the implications of the findings for assessing the benefits of the technologies. 


\section{METHOD}

A telephone survey of system owners was used to gather self-report information related to driver understanding of system functions, capabilities and limitations, as well as reported usage. A total of 480 system owners were recruited using a variety of methods (e.g., mail-outs to lists of vehicle owners, as well as newspaper, magazine, and Internet advertisements, etc.) and administered a 30-minute telephone survey. The survey was intended to gather driver real-world system experiences useful in assessing driver behavioral adaptation (changes in behavior over time), as well as driver acceptance (ease-of-use, effectiveness, desirability, etc.) associated with three in-vehicle devices (Adaptive Cruise Control, Park Aid, and Navigation systems). The sample of 480 valid vehicle owners was heavily represented by males $(67 \%)$ versus females (33\%). The sample included a wide age distribution ranging from 23 to 87 years of age (mean age was 56 years). Over $98 \%$ of the vehicles owned were 2002 or later models, and had been driven an average of 15,606 miles since they were purchased or leased. A substantial percentage of the sample (25\%) had driven the vehicle 7,000 miles or less; the top $25 \%$ of the sample had driven the vehicle over 20,000 miles since it was purchased. Although individuals from 23 states were represented, the vast majority $(92 \%)$ were residents of Colorado, Florida, Illinois, Michigan, New York, Texas, and Virginia.

\section{RESULTS}

\section{ACC System Understanding \& Behavioral Impacts}

ACC system owners reported using the system under a wider range of situations and environments than conventional cruise (including heavy traffic). Experience with the system appeared to increase drivers' willingness to operate the system under extreme or challenging environments. ACC system users with intermediate levels of experience, for example, were somewhat more likely to use the ACC under almost all conditions including degraded conditions (e.g., rain, fog, snow) compared to their counterparts (see Figure 1). Chi Square tests found that drivers with intermediate levels of experience were more likely to use ACC at night than their counterparts (although similar trends occurred for other conditions they were not statistically significant). In some cases, the pattern of use suggests that initially, drivers appear somewhat cautious in their use of the system (limiting the environments in which they choose to operate the system, in many cases), but with additional experience, drivers become more accepting and willing to use the system somewhat indiscriminately (perhaps testing the system limits). One possible interpretation is that as drivers gain familiarity and become experienced with the system's limitations and capabilities, they tend to adopt a more discerning pattern of use based on their previous experiences with the system.

While nearly all drivers reported reading all or some part of the owner's manual relating to the ACC system, many held misconceptions about the functional capabilities of the system. For example, most owners mistakenly believed the ACC system would react to a stopped in-path vehicle, and many were not aware that the system provided an approach warning feature that alerts the driver when manual intervention is required in situations where the system's braking authority is exceeded. Of greater concern are drivers who think that the warning feature operates all the time, when in fact it does not. Over $6 \%$ of drivers in our sample were under the mistaken impression that the approach warning feature is active in their vehicles even when the ACC 
system is disengaged; these individuals are assuming a greater level of protection than the system actually provides. Again, this misinterpretation of the system's capability was not moderated by experience with the system.

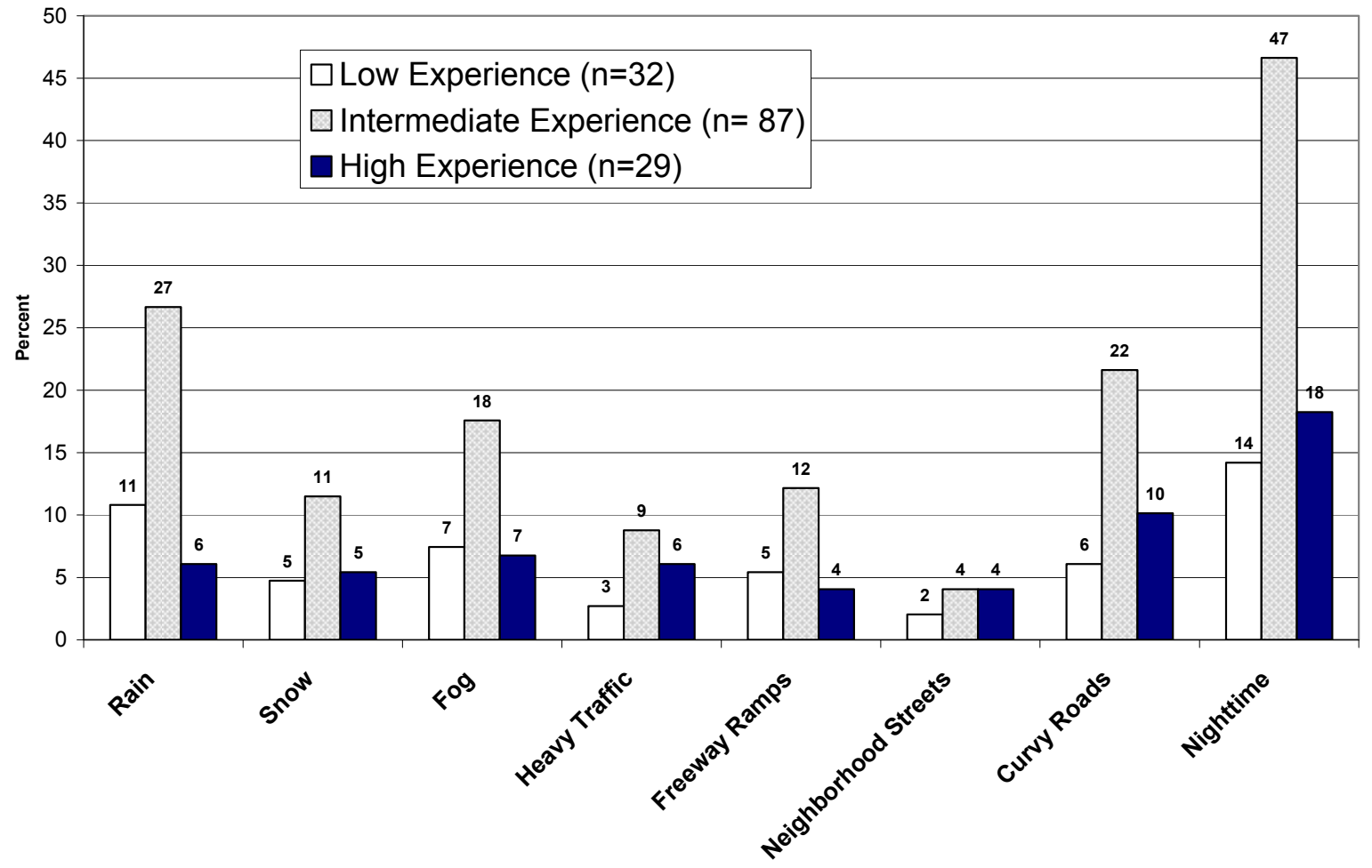

Figure 1. Percent of Drivers Who Report Using the ACC System under Various Operating Conditions as a Function of Experience with System

Data suggest that some drivers appear to modify their behavior as a result of interactions with the ACC system; among these is their use of cruise control, typical following distances, and frequency of lane changes. As shown in Figure 2, almost half of the drivers sampled (48\%) use their ACC more frequently compared to their use of conventional cruise control. The typical following distance to lead vehicles has also increased for $39 \%$ of the drivers sampled; although some drivers (4\%) report adopting shorter headways as a result of using ACC. ACC usage tends to have no appreciable impact on the frequency of lane changes for the majority of drivers (70\%); when modifications do occur, they appear nearly evenly distributed in terms of increasing frequency of lane changes for some $(12 \%)$, and decreasing the frequency for others $(18 \%)$. 


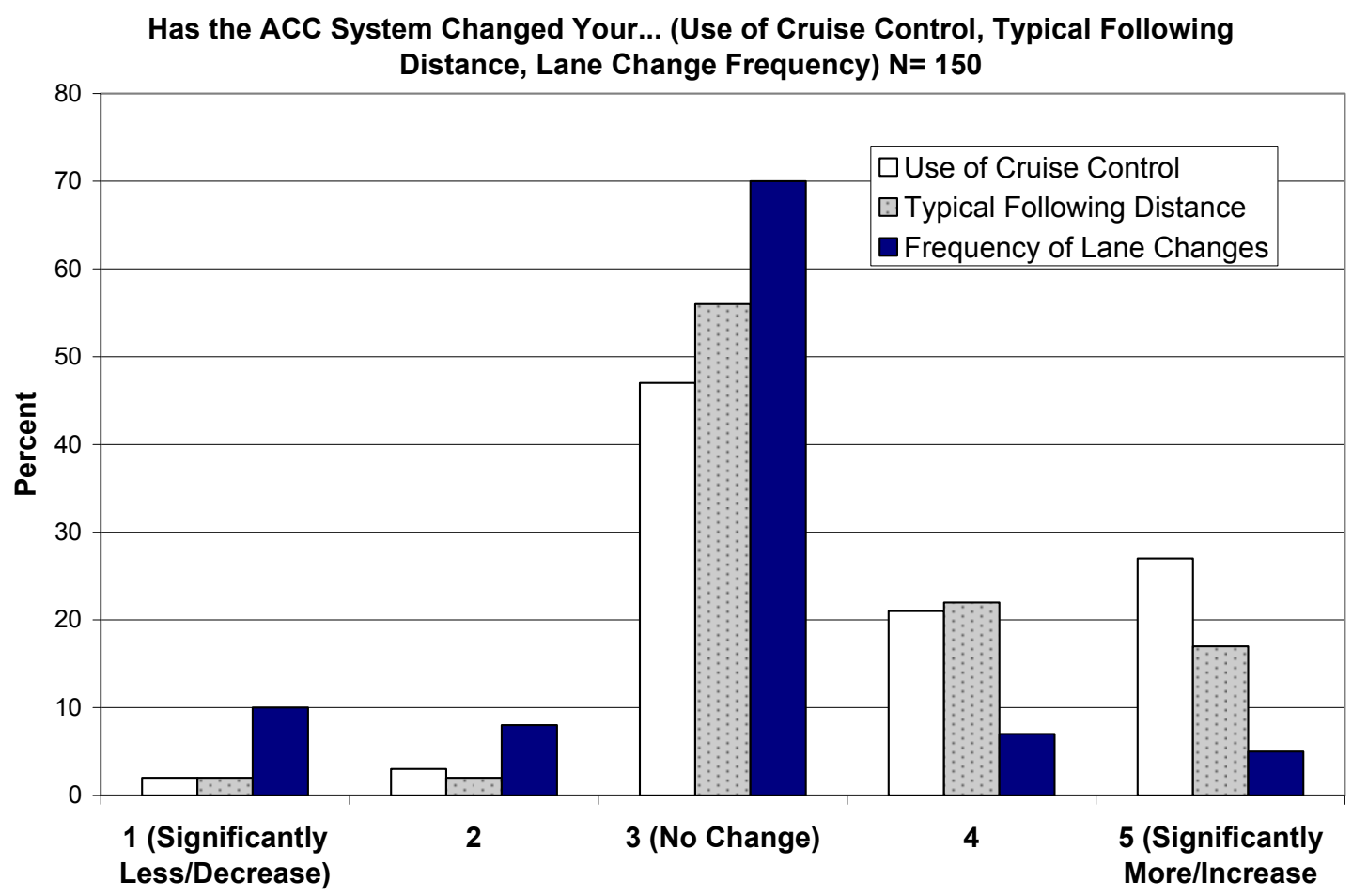

Figure 2. Impacts of ACC Use on Frequency of Use of Cruise Control, Following Distance, and Frequency of Lane Changes

\section{Park Aid System Understanding \& Behavioral Impacts}

Approximately $80 \%$ of owners reported reading some or all of the information relating to the park aid system in their owner's manual. Young drivers were somewhat less likely than their counterparts to read all about the system in manual, while older drivers were somewhat less likely to bypass reading any part of the manual. Of some concern is the finding that a substantial percentage of system owners $(27 \%)$ were unsure of the underlying basis for how the system triggered the warning (distance or a combination of speed and distance). Inexperienced users, those with less exposure to the park aid system, were more likely to incorrectly assume that the system adjusts the timing of the warning based on both speed and distance to the obstacle, rather than using a fixed warning timing based on distance alone. Nearly twice as many "low experience" users $(22 \%)$ were operating under this false assumption compared to their more experienced counterparts $(11 \%)$. The vast majority of drivers were also unaware of the system's functional speed limitations; $67 \%$ believed that the park aid system operates under any speed when backing (most systems only operated at speeds under $6 \mathrm{mph}$ ). Experience with the system also did not appear to improve understanding of the system's functional speed range.

For most drivers (54\%), park aid systems serve as supplements or enhancements to their vision when parking and backing with no reported change to their reliance on direct glances/mirror use. These systems can be very effective; sixty-six percent of park aid system owners report experiencing a situation where the park aid system prevented them form hitting something they had not seen. However, evidence suggests that some drivers may come to over-rely on park aid 
systems, effectively altering their behavior when parking and backing. For example, $20 \%$ of users reported a decreased reliance on the vehicle's mirrors and on direct glances while backing with the system. Thirty-six percent of drivers also indicate that they postpone or delay looking to the rear or glancing in the mirrors when backing with the system engaged, suggesting that some drivers use the system to cue their search behavior. Experience with the system may moderate this behavior to some extent. Shifts in attention (gaze) while backing were reported for drivers who had access to a rear-view camera system with an in-dash display. Presence of the in-vehicle display was reported to change driver's focus while backing in approximately $32 \%$ of the cases. Over one-quarter of these drivers $(28 \%)$ indicated that they relied on the in-dash display more than the mirrors or direct glances, and $4 \%$ relied exclusively on the display while backing. As shown in Figure 3, although drivers of all ages tended to adopt these practices, young drivers in particular may be somewhat more prone to rely on the in-dash camera view exclusively or more than on direct glances or the mirrors while backing (Results of Chi-Square tests were not significant).

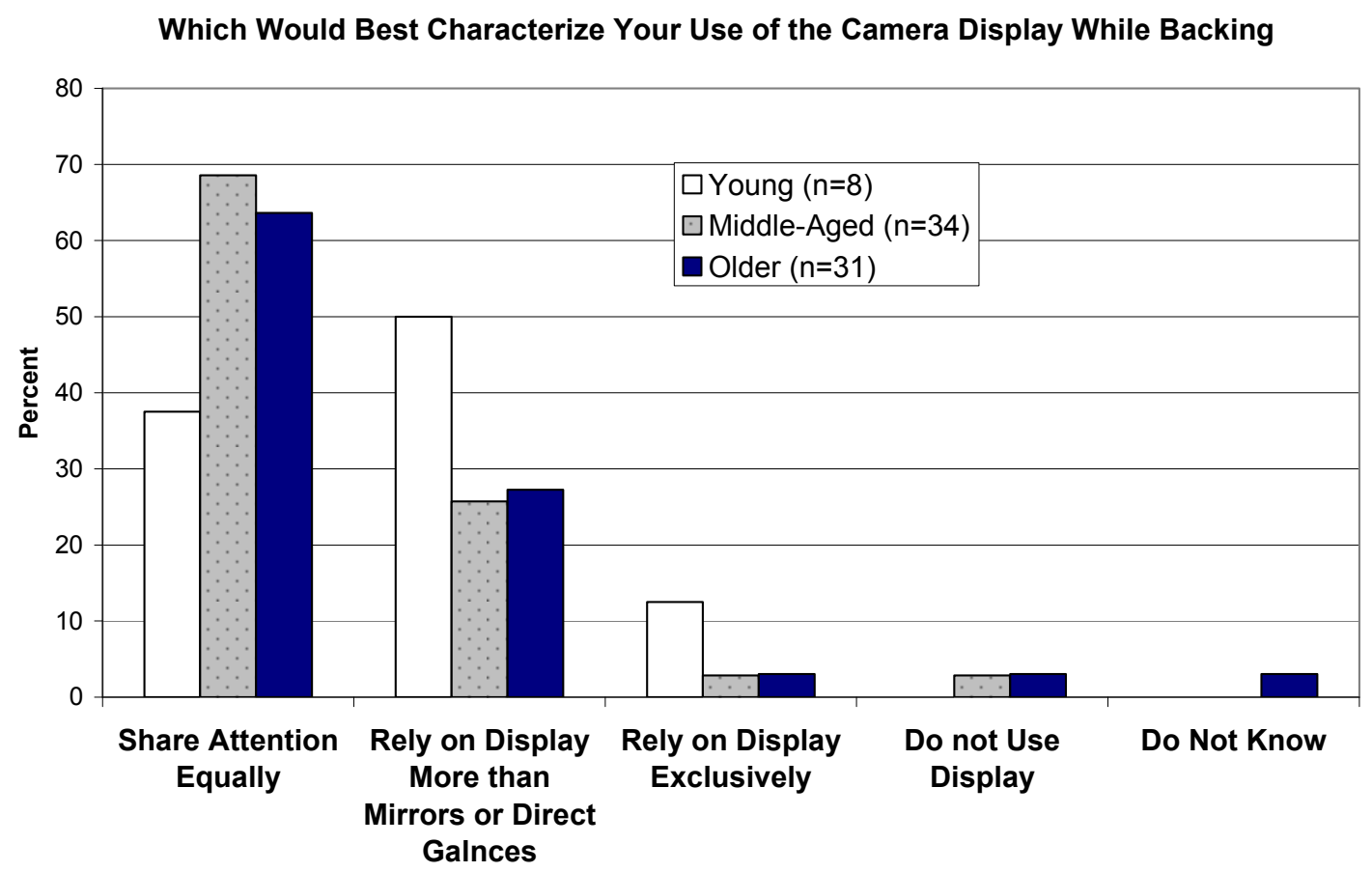

Figure 3. Driver Reliance on In-Vehicle Camera Display as a Function of Age

\section{Navigation System Understanding \& Behavioral Impacts}

Arguably, navigation systems represent one of the most complex systems on the market, incorporating a relatively large number of features and options for configuring displayed information and executing tasks. Many offer a separate owner's manuals or supplement dedicated to providing directions for programming and using the system. Over $93 \%$ of drivers reported reading some or all of the information relating to their navigation system in the owner's manual; older drivers appear much more likely than younger drivers to read information about 
the system in their owner's manual. A surprising percentage of drivers were unsure about even the most basic features of their systems, including the ability to repeat verbal turn instructions or configure the system to provide voice-only turn information (turnoff the visual display). For example, $31 \%$ of system owners were unsure if the system could repeat a verbal instruction if so desired-a common feature on most if not all systems.

Although drivers reported using a range of methods for programming a destination into the navigation system, street address entry was by far the most frequently used destination entry method among drivers; ironically, this method also typically requires the most intensive level of interaction (e.g., button presses, menu levels, etc.). The relatively simpler method of programming via address book was used with much less frequency by drivers (half as frequently as street address). The availability of a navigation system influenced certain types of behaviors, including driver willingness to travel in unfamiliar areas, and the types of roads traveled. This trend was consistent across drivers of all ages, and was not necessarily restricted to older drivers. Some navigation system users report a degraded ability to predict and respond to road hazards when using the system due to an increase in glances away from the road resulting in less scanning of the environment. Nearly one-third of drivers $(32 \%$, or 73 out of 228 drivers) report that they tend to look away from the road more frequently and for longer periods of time when driving with the navigation system. Young drivers were significantly more likely to report that the system causes them to look away from the road more often and for longer periods of time compared to older drivers (see Figure 4); Chi-Square $(4)=9.8, p<.05$. A trend in the data also suggests that more experienced navigation users (high or intermediate experience levels) may reduce their frequency of glances to the environment or mirrors compared to novice system users; $9 \%$ of experienced users indicated reduced scans compared to $3 \%$ of novice users.

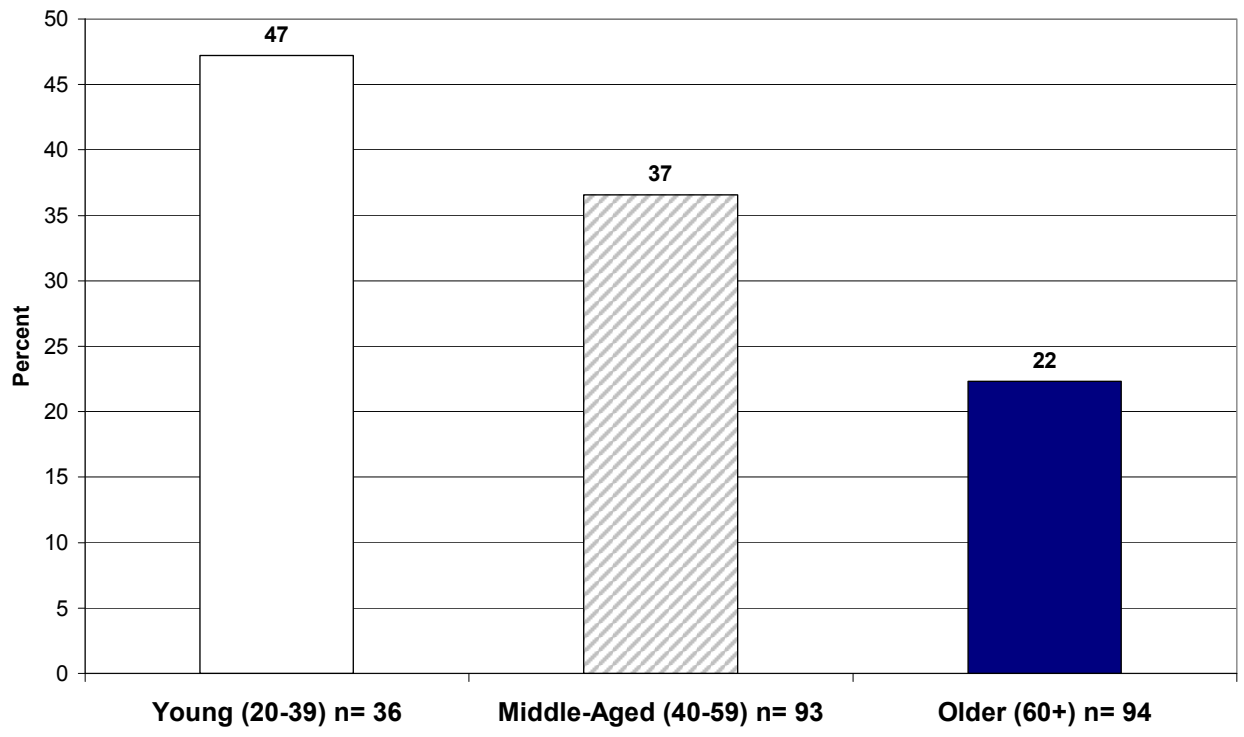

Figure 4. Percent of Drivers Who Report Looking Away From the Road More Frequently and for Longer Periods with Navigation Systems By Age Group 


\section{CONCLUSIONS}

Interviews with owners of advanced in-vehicle systems indicate that these devices are generally well received and liked by drivers. Most drivers feel comfortable using their system within the first few days of use, with system trust and usage increasing over time. Nevertheless, misconceptions are common, suggesting that drivers need to be better educated about the system's capability and limitations. Some form or degree of driver behavioral adaptation was reported to have occurred for each of the systems examined (Llaneras, 2006). Despite access to a wide array of information about their in-vehicle system, responses to knowledge-based questions about the systems themselves suggest that key information was not necessarily acquired or understood by a large number of drivers. Many drivers held misconceptions about the performance capabilities of their advanced systems. For example, 99\% of ACC system owners did not know that the system ignores stopped vehicles. The fact that the system ignores stopped or slow-moving vehicle is available in the owner's manuals for all of the ACC systems reviewed, yet drivers were not aware of this important operational characteristic. Similarly, $41 \%$ of park aid system owners did not know that the system warning is tied solely to the distance to objects and does not take into account their closing speed. This suggests that drivers' mental models of how these systems function and perform do not always match reality, and additional efforts are needed to increase driver understanding of how these systems operate. This is particularly important for safety-related misconceptions.

\section{ACKNOWLEDGMENTS}

This research was sponsored by the Department of Transportation, National Highway Traffic Safety Administration (NHTSA) under contract DTNH22-99-D-07005 while the author was at Westat. The author would like to acknowledge Mike Perel with NHTSA for his support and contributions to the development of the research program and comments regarding the draft paper. Special thanks to Westat's Telephone Research Center for managing and conducting the driver interviews.

\section{REFERENCES}

Llaneras, R.E. (2006). Exploratory Study of Early Adopters, Safety-Related Driving with Advanced Technologies. Final Report Number DOT HS 809 972. U.S. Department of Transportation: National Highway Traffic Safety Administration.

Seppelt, B. and Lee, J. (2007). Making Adaptive Cruise Control Limits Visible. International Journal of Human-Computer Studies, 65(3), 192-205.

Nilsson, L. (1995). Safety effects of adaptive cruise control in critical traffic situations. In: Proceedings of the Second World Congress on Intelligent Transport Systems: 'Steps Forward', III. Yokohama: VERTIS, 1254-1259. 